\title{
Auf dem Weg zu 5G, der 5. Generation des Mobilfunks
}

\author{
H. Malleck OVE, C. Mecklenbräuker
}

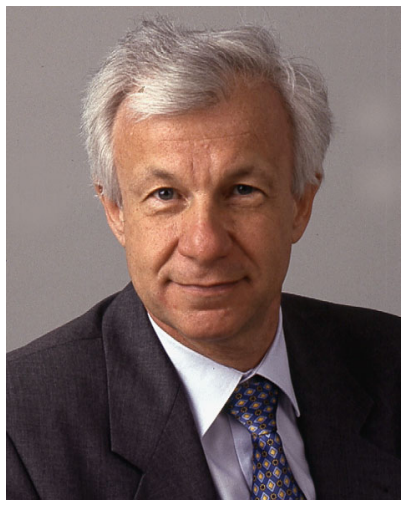

Dipl.-Ing. Dr. Helmut Malleck

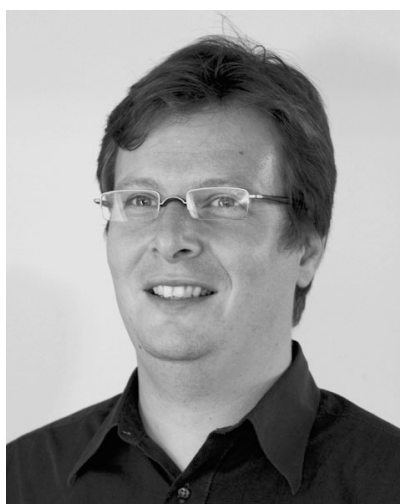

Univ.-Prof. Dipl.-Ing. Dr. Christoph Mecklenbräuker

\begin{abstract}
Liebe Leserin und lieber Leser,

mit $5 G$, der fünften $\mathrm{Ge}$ neration des öffentlichen Mobilfunks, haben wir ein hochaktuelles Thema für die Ihnen vorliegende e\&iAusgabe gewählt, das nach seiner breiten Umsetzung weitreichende Konsequenzen in der Wirtschaft und in unserer Gesellschaft haben könnte.
\end{abstract}

An mobiles Telefonieren haben sich viele von uns seit etwa zwanzig Jahren gewöhnt. Vor zehn Jahren begannen digitale mobile Endgeräte zu unseren ständigen Begleitern zu werden, universell und always on. Parallel dazu entstanden auch neue Anwendungen für den Mobilfunk, wie Mobile Videostreaming und Gaming, Maschine-zuMaschine-Kommunikation, Industrie 4.0 und zuletzt das Internet der Dinge. Mit Mobilfunk-Speziallösungen sollte dem ständig steigenden mobilen Datenvolumen entsprochen werden. Die fünfte Generation des öffentlichen Mobilfunks $5 \mathrm{G}$ steht nun für stark verbesserte mobile Breitbandkommunikation, häufigen Informationsaustausch von Maschine zu Maschine und ultra-zuverlässige Kommunikation mit geringer Latenzzeit.

$5 G$ wird jedoch mehr sein als eine neue Mobilfunk-Generation. $5 \mathrm{G}$ ist ein Quantensprung und soll ab Mitte des kommenden Jahrzehnts in vollem Umfang verfügbar sein - und damit auch schnelle Antworten und hohe Kapazitäten. Standardisierung, Forschung und Entwicklung sowie die Umsetzung von $5 \mathrm{G}$ schreiten weltweit auf Hochtouren voran. Ein allgemeines konzeptmäßiges Verständnis für $5 \mathrm{G}$ und straff vollzogene Standardisierungsaktivitäten führten bereits zu frühen Prototypen, und diese geben zu Optimismus Anlass. Allen Berichten zufolge hat sich $5 \mathrm{G}$ nun von einer Vision zur Realität gedreht.

Für die 5G-Kommunikation werden Spektralbereiche erschlossen, die für die öffentliche Mobilkommunikation bisher noch nicht genutzt werden konnten: der Millimeterwellen-Bereich mit Frequenzen von $6 \mathrm{GHz}$ bis $300 \mathrm{GHz}$. Um sowohl gute Flächendeckung als auch Kapazität in dicht verbauten Gebieten bereitstellen zu können, wird eingeschränkter Empfang, etwa als Folge der Gelände-Topologie oder durch bzw. im Gebäude, entsprechend ausgeglichen. Dies erfolgt unter Einsatz kleiner Funkzellen, mit einer deutlich leistungsfähigeren Antennentechnik, die sich zusätzlich an den Positionen der Endgeräte individuell orientiert, sowie durch separate Übertragungskanäle je Signalrichtung.

Besonders hervorzuheben ist noch die verteilte Topologie der Funkzellen-Rechner im 5G-Mobilnetz mit zellenübergreifendem Cloud-Computing. Auf der gemeinsamen physikalischen 5G-Infrastruktur können auch mehrere logisch unabhängige Netze eingerichtet werden, die entsprechend den spezifischen Erfordernissen der Anwendungen - ultraschnell, sehr niedere Latenzfähigkeit etc. - konfiguriert sind. Solche 5G-Anwendungen, die sich der gemeinsamen 5G-Kommunikationsbasis bedienen, sind beispielsweise autonomes Fahren, drahtlos operierende (humanoide) Roboter und Drohnen bzw. 5G-Anwendungen zur Virtual Reality. Dafür muss 5G hohe Kapazitäten bereitstellen, derer es für die perfekte Immersion hochauflösender Bilder bedarf. $5 \mathrm{G}$ wird also mehr als nur eine neue Mobilfunk-Generation sein

\section{Originalarbeiten zum Themenschwerpunkt}

Das vorliegende Themenheft umfasst sechs Originalarbeiten. Einleitend wird die 5G-Standardisierung bei ETSI behandelt. Es folgen Beiträge zu 5G-Forschungs- und Innovationsprojekten sowie den Anforderungen zukünftiger 5G-fähiger Anwendungen. Daran schließt ein Beitrag des Österreichischen Patentamts an, denn gerade bei technologischen Umbrüchen, aktuell mit Digitalisierung und Breitbandkommunikation im 5G, kommt dem Schutz von geistigem Eigentum durch Patente oder Marken besondere Bedeutung. Als letzte Originalarbeit finden Sie einen historischen Überblick zu öffentlichen Mobilfunksystemen. Darüber hinausgehende Themen zu $5 G$, wie etwa zum 5G-Systemkonzept, zu Anforderungen und Positionierung von $5 \mathrm{G}$ im Frequenzspektrum, zu Zugangstechnologien für $5 \mathrm{G}$ im Funknetz bzw. wie der öffentliche Sektor optimale Rahmenbedingungen für $5 \mathrm{G}$ schaffen kann, wurden beim heurigen IT-Kolloquium abgehandelt. Siehe dazu auch die Nachlese in e\&iAusgabe 4-5, 2018. ${ }^{1}$

- Luis Jorge Romero stellt in seinem Beitrag "5G Standardization in ETSI" die 2015 in neuem Format bei ETSI begonnene, mit 3GPP harmonisierte 5G-Standardisierungsarbeit vor. Über die Standardisierung der Basiskomponenten von 5G-Netzwerken hinaus berei-

${ }^{1}$ Malleck, H. (2018): IT-Kolloquium 2018: Auf dem Weg zu 5G. e\&i, H. 4/5 (2018), S. a17 f.

Malleck, Helmut, Arbeitskreis für technische Koordination für öffentliche

Kommunikationsnetze und -dienste (AK-TK), Postfach 147, 1006 Wien, Österreich

(E-Mail: ak-tk@oefeg.at); Mecklenbräuker, Christoph, Institute of Telecommunications, Technische Universität Wien, Gußhausstraße 25-25a, 1040 Wien, Österreich 
tet ETSI auf Basis von virtualisierten Netzfunktionen und Software Defined Networking neue Netzarchitekturen vor, zeitlich abgestimmt mit den Releases 15 und 16 usw. Abschließend wird 5GStandardisierung in Bezug auf Public Safety, Factory Automation, Railway Communications, Vehicle to Vehicle, Drones and Airborne Wireless Access Points kurz erläutert.

- Der Beitrag von Thomas Zemen „Wireless 5 G ultra reliable low latency communications - European and Austrian research initiatives" gibt einen aktuellen Überblick von auf EU-Ebene, in Österreich und am Austrian Institute of Technology (AIT) laufenden Forschungsprojekten zu 5G. Die Architektur zukünftiger 5G-Systeme, ihre Anwendungsfälle und wichtige physikalische Funktechnologien werden beschrieben. Weiters wird der aktuelle Stand der 5GForschung in Europa mit dem EU-Forschungsrahmenprogramm "Horizont 2020" vorgestellt. Darüber hinaus wird ein Überblick der Forschungsaktivitäten in Österreich präsentiert und abschlieBend der 5G-Forschungsschwerpunkt am AIT im Bereich der hochzuverlässigen 5G-Funkkommunikation mit niedriger Latenzzeit beschrieben.

- Stefan Schwarz et al. geben im Beitrag "Dependable wireless connectivity: insights and methods for $5 G$ and beyond" einen Überblick über die im Christian Doppler Labor entwickelten Methoden, um die Zuverlässigkeit von Mobilfunksystemen zu verbessern, sowie über die daraus resultierenden Weiterentwicklungen von Mobilfunktechnologien. Neuartige Anwendung von drahtlosen Übertragungssystemen, wie zum Beispiel der Austausch verkehrssicherheitsrelevanter Informationen in der Fahrzeugkommunikation oder die drahtlose Steuerung von Robotern und Drohnen, verlangen nach einem sehr hohen Grad an Zuverlässigkeit. Dazu sind derzeit die Arbeiten an der fünften Mobilfunkgeneration, mit sehr geringen Latenzzeiten und hoher Ausfallsicherheit, in vollem Gange.

- Maria D. Pérez Guirao et al. nehmen sich in ihrem Beitrag "Audiovisual networks and the road to $5 \mathrm{G}$ - on standardization, role models and spectrum access" der professionellen audio-visuellen Medienproduktionen an und diskutieren Standardisierungsaspekte rund um flexible Modelle, die eine entsprechende Anwendung innerhalb 5 G möglich machen. Dabei wird die Umsetzung einer geeigneten Funkschnittstelle vorausgesetzt. Sehr anschaulich werden die Erfordernisse eines leistungsfähigen 5G-Supports bei zukünftigen professionellen audio-visuellen Medienproduktionen dargelegt und Inputs für erforderliche Standardisierungen gegeben.

- Christian Seyringer et al. zeigen in ihrem Beitrag „Der ungesicherte Goldschatz - Start-ups und KMU denken bei IP an das Internet und nicht an ihr geistiges Eigentum (Intellectual Property)", dass von Jungunternehmen auf den Schutz geistigen Eigentums durch Patente oder Marken nur allzu oft vergessen wird. Das Österreichische Patentamt (ÖPA) bietet eine Vielzahl von Service- und kostenlosen Beratungsleistungen, um sich vor dem Kopieren von Produkten zu schützen. Dieser Beitrag gibt dazu einen Überblick, ergänzt um wertvolle Tipps der ÖPA-Experten.

- Der Beitrag von Helmut Malleck „Technologische Evolution der auf GSM basierenden öffentlichen Mobilfunksysteme" gibt dem technisch interessierten Leser einen Überblick über die technologische Evolution der auf dem Mobilfunkstandard Global System for Mobile Communication (GSM) basierenden Mobilfunksysteme. Es werden Netzarchitekturen, Übertragungsverfahren und Dienste kurz vorgestellt und auf die weitergehende Literatur in der Standardisierung verwiesen.

\section{Weiterführende Themen in den Berichten}

Der abschließende Bericht im vorliegenden Themenheft soll Ihnen ein weiterführendes Thema, das längerfristig auch vom 5GBreitbandausbau profitieren würde, näherbringen.

- Gernot Fischer und Matthias Pistrol benennen in ihrem Beitrag "Digitalradio im Standard DAB+ in europaweitem Aufwind" die Gründe für eine Umrüstung der gesamten Hörfunkterrestrik auf DAB+. Dem Neuaufbau der Senderinfrastruktur und der Anschaffung neuer Empfangsgeräte stehen u. a. zeitversetzter ondemand Medienkonsum mit Rückkanal, Bündelung vieler Radioprogramme mit optimiertem Ressourcen- und Energieeinsatz $u$. v. m. gegenüber. Nahezu alle europäischen Länder haben DAB+, zumindest bereits regional, in Betrieb gesetzt.

Um mit der Umsetzung von $5 \mathrm{G}$ die sich daraus ergebenden Vorteile nutzbar zu machen, müssen drastische Verbesserungen in der zellularen Netzarchitektur vorgenommen werden. Erste Schritte auf diesem Weg sind mancherorts bereits gesetzt worden. Ob vorab oder auch zusätzlich einzelne Industrien separate 5G-Netze als hochwertigen Ersatz für aktuelle W-LAN-Systeme errichten, wäre eine Option, die es abzuwarten gilt.

Eine spannende Lektüre der Beiträge zu diesem Themenschwerpunkt wünschen Ihnen

\section{Helmut Malleck}

und

Christoph Mecklenbräuker 